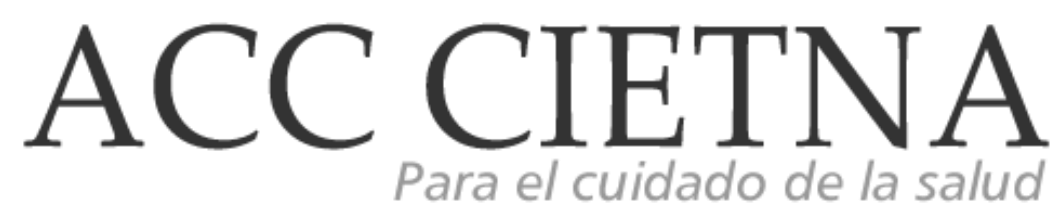

https://doi.org/10.35383/cietna.v7i1.352

\title{
La enseñanza-aprendizaje del proceso de atención de enfermería en estudiantes del noveno ciclo: una experiencia enriquecedora
}

\author{
Quispe Ramos Luz Estefany', Mogollón Torres Flor de María
}

\begin{tabular}{l}
\hline INFORMACIÓN DEL ARTÍCULO \\
\hline Historia del artículo: \\
Recibido el 15 de mayo de 2020 \\
Aceptado el 15 de junio de 2020 \\
\hline Palabras clave: \\
Experiencia \\
Enseñanza \\
Aprendizaje \\
Enfermería
\end{tabular}

\section{RESUMEN}

Introducción: El Proceso de Atención de Enfermería (PAE), es una herramienta básica en esta profesión, este ofrece una estrategia ordenada para resolver problemas y suministrar cuidados de enfermería, además es un instrumento a usarse a lo largo de la carrera de enfermería, por ello, es vital conocer cómo se está dando el proceso de enseñanza-aprendizaje. Objetivo: Describir, analizar y comprender las experiencias en la Enseñanza-Aprendizaje del PAE en estudiantes del noveno ciclo. Métodos: Estudio cualitativo, de abordaje estudio de caso, cuya muestra fueron 16 estudiantes y 14 docentes de una Escuela de Enfermería; el muestreo se realizó por conveniencia y el tamaño se determinó por saturación y redundancia; los datos se recolectaron mediante una guía de entrevista semiestructurada, validada por juicio de expertos y por piloto; el análisis de datos fue mediante el análisis de contenido; se consideró siempre criterios de rigor científico y éticos. Resultados principales: Cinco categorías: (1) Enseñanza-aprendizaje del PAE: uso de la taxonomía NANDA, NIC, NOC y estrategias de enseñanza, (2) Experiencias en la aplicación del PAE en el contexto hospitalario y comunitario, (3) Experiencias en la evaluación de la enseñanza aprendizaje del PAE, (4) Adquisición de habilidades en el proceso de enseñanza-aprendizaje del PAE y (5) Necesidad de estandarizar criterios en la enseñanza del PAE. Conclusión general: No existe uniformidad en la enseñanza del PAE, lo cual genera dificultad en su aprendizaje; las mismas que influyen en la aplicación, ya sea en el ámbito hospitalario o comunitario; es evaluado desde la elaboración del informe hasta el acto de sustentación y su aplicación genera

'Licenciada en Enfermería. Egresada de la Escuela de Enfermería de la Universidad Católica Santo Toribio de Mogrovejo, Chiclayo, Perú. Email: luz.qr.13@outlook.com. ORCID: https://orcid.org/0000-0002-9976-2931

2Magister en Ciencias de Enfermería. Especialista en Enfermería Materno Infantil con mención en Neonatología. Docente de la Escuela de Enfermería USAT. Enfermera asistencial en el Centro Materno Infantil Toribia Castro Chirinos, Lambayeque, Perú. Email: fmogollon@usat.edu.pe. ORCID https://orcid.org/0000-0002-2605-546X 
habilidades cognitivas y sociales en los estudiantes; asimismo, se reconoce como necesidad prioritaria la estandarización de la enseñanza del PAE.

Teaching-learning the nursing care process in students of the ninth cycle: an enriching experience

ABSTRACT

Keywords:

Experience

Teaching

Learning

Nursing

Introduction: The Nursing Care Process (PAE) is a basic tool in this profession, it offers an orderly strategy to solve problems and provide nursing care, it is also an instrument to be used throughout the nursing career, for it is vital to know how the teaching-learning process is taking place Objective: Describe, analyze and understand the experiences in the PAE Teaching-Learning in students of the ninth cycle. Methods: Qualitative study, of case study approach, whose sample was 16 students and 14 teachers of a School of Nursing; the sampling was done for convenience and the size was determined by saturation and redundancy; the data were collected through a semistructured interview guide, validated by expert judgment and by pilot; the data analysis was through content analysis; criteria of scientific and ethical rigor were always considered. Main Results: Five categories: (1) Teaching-learning of the PAE: use of the NANDA taxonomy, NIC, NOC and teaching strategies, (2) Experiences in the application of the PAE in the hospital and community context, (3) Experiences in the evaluation of the teaching - learning of the PAE, (4) Acquisition of skills in the teaching-learning process of the PAE and (5) Need to standardize criteria in the teaching of the PAE. Overall conclusion: There is no uniformity in the teaching of the PAE, which generates difficulty in their learning; the same that influence the application, either in the hospital or community setting; it is evaluated from the preparation of the report until the support act and its application generates cognitive and social skills in the students; Likewise, the standardization of teaching of the PAE is recognized as a priority need.

\section{Introducción}

La enfermería es ciencia y arte, que se relaciona con cuestiones físicas, psicológicas, sociales, culturales y espirituales del individuo. En tal sentido, una herramienta básica de la enfermería es el Proceso de Atención de Enfermería (PAE), este ofrece una estrategia ordenada y lógica para la resolución de problemas y tiene como objetivo suministrar los cuidados de enfermería, de tal manera que las necesidades de atención del paciente se cubran por completo y de manera efectiva'.

Existe la idea, que el proceso consiste simplemente en emitir un diagnóstico de enfermería o un plan de actividades ${ }^{2}$. Alfaro, lo define como "un método sistemático y organizado para administrar cuidados de enfermería individualizados" ya que cada persona responde de forma distinta ante una alteración real o potencial"3. El proceso de enseñanzaaprendizaje universitario, debe estar dirigido a 
conseguir un aprendizaje profundo en los estudiantes 4 .

En la Facultad de Enfermería de la Pontificia Universidad Javeriana, en Colombia, la aplicación del PAE se utiliza como herramienta pedagógica y facilitadora del aprendizaje en cada uno de los semestres de la carrera. Para ello, el docente de enfermería, ofrece una formación integral al estudiante, lo que le permite desarrollar el aprendizaje $y$ adquirir habilidades para diligenciar el PAE, el cual se inicia con el desarrollo de la guía de valoración del paciente, por medio de los patrones funcionales de Gordon, que les facilitan al docente y al estudiante, trabajar de forma organizada, científica e individualizada, estimulando el pensamiento crítico y reflexivo 5 .

Dolores ${ }^{6}$ en un estudio encontró que durante los primeros ciclos el proceso de enseñanzaaprendizaje del PAE no era coherente, puesto que, a pesar que la enseñanza teórica era buena y que los resultados de los estudiantes eran óptimos en los diferentes casos teóricos, en la parte práctica, los estudiantes, expresaban que la aplicación del PAE era parcial o poca en la atención al paciente, por lo tanto, no se lograba una óptima aplicación.

En Chiclayo, a nivel de universidades, tanto públicas como privadas, no existe un patrón definido sobre el proceso de enseñanzaaprendizaje del PAE, a pesar de que todas intenten estandarizar la enseñanza, según la taxonomía NANDA, NIC, NOC, cuyas siglas significan NANDA: Asociación Norteamericana de Diagnósticos de Enfermería (North American Nursing Diagnosis Association); NIC: Clasificaciones de Intervenciones de Enfermería (Nursing Interventions Classification) y NOC: Clasificación de Resultados de Enfermería (Nursing Outcomes Classification); hay algunas variantes.

Siendo que el estudiante del noveno ciclo, es el sujeto que ha transitado ya por las diferentes metodologías de enseñanza del PAE durante su formación profesional y a pesar que en ese ciclo académico ya no recibe la enseñanza del mismo, y asumiendo que el estudiante en este nivel lo conoce y lo aplica correctamente, es que se realizó la investigación con este grupo de estudiantes.

En este marco de referencia, las investigadoras se han enfocado en describir, analizar y comprender las experiencias en la Enseñanza-Aprendizaje del Proceso de Atención de Enfermería en estudiantes del noveno ciclo de una universidad privada, desde las vivencias de las estudiantes y docentes, en el contexto académico.

Esta investigación, se justificó porque en el Perú, la aplicación del PAE obedece a una exigencia legal establecida en la Ley del Trabajo de la Enfermera(o) (Ley N ${ }^{\circ} 27669$ - capítulo II, artículo 7), que establece que corresponde a la enfermera brindar cuidado integral de Enfermería basado en el PAE, el cual será registrado obligatoriamente en la historia clínica. Además, se justifica porque actualmente, a nivel nacional y local no hay investigaciones relacionadas directamente con el objeto de estudio.

\section{Metodología}

La presente investigación fue de tipo cualitativa $^{7,8}$, ya que se requirió indagar a los sujetos en su relación íntima con el objeto de estudio: las experiencias en el proceso de enseñanza-aprendizaje del PAE, analizándolo en su propio contexto, permitiendo así una mejor comprensión. El abordaje de investigación fue, estudio de caso ${ }^{9}$, el cual se llevó a cabo la Escuela de Enfermería de una Universidad Católica, situada en la ciudad de Chiclayo, provincia de Lambayeque, al norte del Perú; promovida y administrada por el obispado de la diócesis de Chiclayo. Reconocida por su excelencia educativa y por ser la primera universidad de la región Lambayeque en tener carreras acreditas por 
el SINEACE y licenciada oficialmente por la SUNEDU en el 2018.

La muestra fue en total 30; de los cuales, 16 fueron estudiantes del noveno ciclo de la escuela de enfermería USAT, cuyas edades oscilaron entre los 20 y 28 años, el $94 \%$ de sexo femenino y el $6 \%$ masculino, el $62 \%$ de condición regular y el $38 \%$ irregular; quienes fueron seleccionados considerando algunos criterios de inclusión, lo que permitió una muestra homogénea e idónea para explorar fenómeno en profundidad ${ }^{10}$ : Así, se incluyó a estudiantes que hayan estudiado desde el primer al noveno ciclo en la Escuela de Enfermería, independientemente de su estado académico (alumno regular o irregular) y que estén matriculados en el noveno ciclo del semestre en el que se llevó a cabo la investigación (2017-II); Excluyéndose a estudiantes de traslado externo, estudiantes que participaron en la prueba piloto y a los estudiantes que pertenecieron al PIMEU (Plataforma Internacional de Movilidad Estudiantil universitaria).

También, se entrevistaron a 14 docentes involucrados en el proceso de enseñanzaaprendizaje del PAE, con el fin de obtener una mayor amplitud de datos y una mayor visión del objeto de estudio, con dichos resultados se realizó, la triangulación de sujetos 10. Los años de experiencia docente de las enfermeras entrevistadas oscilaron entre 8 a 25 años; el $100 \%$ cuenta con estudios de especialidad o postgrado; el 93\% (13) trabaja en el área asistencial y docente al mismo tiempo, y solo el $7 \%$ (1) en el área docente. Los docentes cumplieron con los siguientes criterios de inclusión: docentes de teoría y práctica de las asignaturas de especialidad del segundo al sétimo ciclo, con una experiencia docente mínima de seis meses, excluyendo a dos docentes que participaron en la prueba piloto.

El muestreo, fue no probabilístico ${ }^{10}$, por conveniencia y el tamaño de la muestra se determinó por saturación y redundancia ${ }^{11}$, dejando de aplicar el instrumento cuando no se encontró datos nuevos.

Los datos se recolectaron mediante la entrevista semi-estructurada7, abierta a profundidad; esta permitió obtener de manera amplia, las experiencias de los estudiantes y docentes sobre el objeto de estudio. El instrumento fue una guía de entrevista, elaborada por las investigadoras, y validada por juicio de expertos (docentes investigadores expertos en la temática y en investigación cualitativa, por más de 8 años) y por prueba piloto ( 2 estudiantes y 2 docentes en el mismo escenario de investigación) ${ }^{11}$.

Como primer paso, se realizó la inscripción del proyecto de investigación en el catálogo de Tesis de la Escuela de Enfermería, luego se presentó al Comité Metodológico de dicha escuela conformado por dos investigadores externos al estudio, quienes aplicaron una lista de cotejo y dejaron sugerencias, lo que permitió mejorar la calidad de la investigación, en seguida se logró la aprobación del Comité de Ética en Investigación de la Facultad de Medicina, con Resolución $\mathrm{N}^{\circ}$ 147-2017-USAT-FMED. Posteriormente, se solicitó el permiso en la Escuela de Enfermería para la ejecución del proyecto y la asignación de un ambiente para ejecutar la entrevista. Una vez identificados a los participantes en la Oficina de asuntos académicos de dicha escuela, se procedió a un primer contacto con cada uno de ellos de manera personal y alguna vía telefónica, para informar el propósito de la entrevista y para la programación de la fecha y hora de la entrevista según su disponibilidad.

Las entrevistas fueron ejecutadas en la sala de docentes de la escuela y fueron transcritas en Word después de realizada cada una, a fin de mejorar el proceso de recolección de datos; luego, se regresó al escenario las veces necesarias hasta que se completó el tamaño de la muestra, la cual fue determinada por los criterios de saturación y redundancia. Además, para dar mayor validez a los resultados, se realizó el chequeo de participantes ${ }^{10}$, de manera 
personalizada, lo que permitió corroborar los relatos y profundizar algunos aspectos poco abordados en la entrevista; Luego de todo ello, se procedió al procesamiento de la información. La base de datos fue guardada, previo consentimiento y autorización de los sujetos por un periodo de dos años, a fin de que sirvan como fuente de verificación de la investigación.

El análisis de datos se realizó mediante el análisis de contenido'1, el cual consistió en descomponer el corpus de los relatos, extraer las unidades textuales por áreas temáticas; en seguida identificar los elementos de significado; para proceder a la agrupación por convergencias de indicadores, obteniéndose así categorías empíricas, las cuales, al confrontarse con el marco teórico, se construyeron las categorías de estudio, relacionados al objeto de investigación.

Durante el desarrollo de la investigación se aplicaron los principios de la ética personalista ${ }^{12}$ : valor fundamental de la vida humana, respetando en todo momento las diversas opiniones de los participantes, protegiendo su identidad con códigos; principio de libertad y responsabilidad, toda vez que los datos se recolectaron una vez aprobado el proyecto por el Comité de Ética en investigación de la universidad, con el previo permiso de la Dirección de escuela y el consentimiento informado de los sujetos. Por otro lado, se aplicó los criterios de rigor científico ${ }^{13}$ como la credibilidad, la auditabilidad y la transferibilidad o aplicabilidad.

\section{Resultados, análisis y discusión}

\section{CATEGORÍA I: ENSEÑANZA-APRENDIZAJE DEL PAE: USO DE LA TAXONOMÍA NANDA, NIC, NOC Y ESTRATEGIAS DE ENSEÑANZA}

El PAE, desde ya hace muchos años atrás se ha implementado como herramienta de cuidado. Este, es transmitido durante toda la formación de los profesionales de enfermería, con el objetivo de que cuando sea profesional, brinde un cuidado con fundamentación científica, y por ende de calidad ${ }^{14}$. Para Doenges, el PAE ofrece una estrategia ordenada y lógica para la resolución de problemas y tiene como objetivo suministrar los cuidados de enfermería, de tal manera que las necesidades de atención del paciente se cubran por completo y de manera efectiva ${ }^{\text {. }}$.

El PAE comprende actualmente un código universal y estandarizado, y son tres elementos: NANDA-NIC-NOC, cuyas siglas significan NANDA: Asociación Norteamericana de Diagnósticos de Enfermería (North American Nursing Diagnosis Association); NIC: Clasificaciones de Intervenciones de Enfermería (Nursing Interventions Classification) y NOC: Clasificación de Resultados de Enfermería (Nursing Outcomes Classification); los que componen el lenguaje estandarizado de la enfermería (LEE). El empleo de un LEE evita que los problemas detectados por la enfermera en el paciente y la familia, se describan mediante un estilo «libre» 15 .

Actualmente, la Escuela de Enfermería de la Universidad donde se realizó la investigación, mantiene un lenguaje estandarizado $y$ capacitaciones eventuales para reforzar este LEE, pero son las docentes, quienes no han acatado en su totalidad este lenguaje estandarizado. Lo mencionado se puede evidenciar en el siguiente discurso:

\section{“...cuando a nosotros nos empezaron a enseñar el PAE, para mí se me hizo un poco tedioso porque uno recién estaba aprendiendo, es algo tedioso también aprender el NANDA...para mí se volvía a veces muy estresante, y casi siempre lo veía muy difícil..." (E1).}

La mayoría (10/16) de los estudiantes de enfermería que participaron en el estudio, refieren que el proceso de aprendizaje y el uso de las taxonomías NANDA, NIC, NOC fue difícil, siendo las causas de esta dificultad el aprendizaje del manejo de la taxonomía, la falta 
de uniformidad en la enseñanza del PAE y el proceso adaptativo a un aprendizaje nuevo, cabe resaltar que en este porcentaje se encuentran las estudiantes de condición irregular (repitentes).

Por el contrario, un mínimo de los estudiantes entrevistados $(2 / 16)$ refieren, que el proceso de aprendizaje del PAE fue fácil, siendo ello posible por la adecuada instrucción que brindó la docente a cargo de la enseñanza del PAE; en tanto los demás $(4 / 16)$ de condición estudiantil regular, refieren que en el proceso de enseñanza del PAE les explicaron detalladamente el uso de la taxonomía, las etapas y la aplicación, sin referir facilidad o dificultad en el aprendizaje del mismo.

A ello, se pueden agregar las afirmaciones de las docentes a cargo de la enseñanza del PAE, quienes también reconocen la dificultad en el aprendizaje:

"...no hay uniformidad de criterios para enseñar a los estudiantes, usan distintos tipos de formato, de metodologías, la valoración algunos la realizan por necesidades, otros por dominios, otros por patrones funcionales, eso causa confusión en el estudiante, y a veces en vez de ayudarlo, uno lo confunde, sobre todo en la aplicación de las taxonomías NANDA, en el que a veces ni las mismas profesoras están capacitadas en el uso de esas taxonomías, y a veces hay una resistencia a asumir el estudio de la taxonomías..." (D4).

La mayoría (10/14) de las docentes, afirman que el proceso de enseñanza del PAE es difícil para con las estudiantes, esto por la falta de uniformidad en la didáctica y por el proceso de adaptación que las estudiantes atraviesan al adquirir un nuevo conocimiento, cabe resaltar que estas docentes cuentan con experiencia en esta área mayor a 8 años y por tanto, experiencia en la enseñanza del PAE; Por otro lado, las 4/14 restantes, afirman que el PAE es un método científico que ayuda al estudiante a respaldar el cuidado que brinda, durante su formación $y$ luego cuando se profesional, sin manifestar facilidad o dificultad en la enseñanza del mismo.

Cachón ${ }^{15}$, en su investigación develó que las enfermeras de cuidados intensivos, tienen dificultad en el momento de aplicar el lenguaje estandarizado en la práctica clínica, refiriendo que para el uso de planes de cuidados estandarizados basados en el LEE es necesario emplear mucho más tiempo, requieren tiempo de formación y un lenguaje específicamente enfermero, provoca dificultades en la comunicación con otros profesionales que no sean enfermeras.

Por otro lado, en el presente estudio se encontró que la enseñanza del PAE se desarrolla haciendo uso de una variedad de estrategias, estas son, el conjunto de decisiones que toma el docente para orientar la enseñanza con el fin de promover el aprendizaje de sus alumnos. Se trata de orientaciones generales acerca de cómo enseñar un contenido disciplinar ${ }^{5}$. A continuación se relatan los discursos obtenidos en la investigación relacionados con las estrategias de enseñanza del PAE:

“...nos enseñaban mediante casos clínicos que las docentes nos brindaban, te daban una situación problema, y tenías que desarrollar cada etapa del PAE, nos hacían trabajar por asociaciones grupales que se formaban dentro del aula. Primero las docentes exponían con power point, luego se finalizaban con las exposiciones por parte de las estudiantes..." (E4).

Referente a las estrategias de enseñanza, se obtuvo que más de la mitad (9/16) de los estudiantes refieren que la enseñanza del PAE incluye la asignación de casos clínicos de manera grupal durante las sesiones teóricas; y el resto (7/16) afirman, que la enseñanza del PAE, fue realizada mediante la asignación de casos clínicos, de manera individual, durante las prácticas clínicas. 
A esto, se añade los discursos referidos por las docentes involucradas en el proceso de enseñanza-aprendizaje del PAE:

“...la metodología está basado en el diálogo personalizado con cada estudiante, ellos ya traen un conocimiento sobre el PAE, entonces lo único que nosotras vamos a hacer es orientarlos, asesorarlos, para que puedan completar sus datos y puedan elaborar un buen PAE...aunque también algunos para avanzar la asesoría se da virtual mediante el correo..." (D2).

Las estrategias usadas por las docentes de enfermería en la transmisión de la enseñanza del PAE, incluyen la asignación de casos clínicos tipos en el aula, generalmente de manera grupal, dato que refieren $7 / 14$ de las docentes entrevistadas y $9 / 16$ estudiantes; mientras que el otro porcentaje $(7 / 14)$ de las docentes refieren que la enseñanza del PAE se realiza, además con la asignación de casos clínicos individuales en la práctica clínica, donde algunas docentes refuerzan la enseñanza del PAE de manera personalizada con cada estudiante asignada, que en promedio son cinco por rotación.

Al respecto, la Dirección de Investigación y Desarrollo Educativo de Monterrey ${ }^{16}$, refiere que la asignación de casos clínicos consiste precisamente en proporcionar una serie de casos que representen situaciones problemáticas diversas de la vida real para que se estudien y analicen. De esta manera, se pretende entrenar a los alumnos en la generación de soluciones.

Una vez que estudiantes y docentes, manifestaron sus experiencias en cuanto a las metodologías de enseñanza-aprendizaje del PAE, se centraron en describir cuál de las etapas del PAE, es la más fácil y la más difícil de enseñar y aprender; es así, que la etapa de valoración es considerada como la más fácil, tanto por docentes, como por estudiantes; cabe resaltar también, que según Accornero, la valoración, es la etapa donde la enfermera adquiere datos, identifica las necesidades o los problemas que se presentan en el inidividuo. ${ }^{17} \mathrm{Al}$ respecto, los estudiantes manifiestan:

“...la valoración es la etapa más fácil, porque te daban unos formatos de valoración, ya sea en el ámbito hospitalario o comunitario, entonces tú ibas preguntando, recogiendo y clasificando los datos subjetivos, objetivos, históricos, los antecedentes, y eso para mi fue más fácil, que las demás etapas del PAE..." (E9).

La mitad (8/16) de los estudiantes refieren, que la etapa del PAE considerada como la más fácil de aprender y aplicar es, la valoración, la misma que se realiza mediante guías de recolección de datos, con diversas fuentes y técnicas de obtención de información. 7/16 refieren, que la etapa más fácil de aprender y aplicar es la etapa de diagnóstico, señalando que presentaban facilidad en la validación de datos significativos del paciente con las características definitorias de la NANDA, permitiendo que la formulación de diagnósticos de enfermería sea más sencilla; y solo $1 / 16$ afirman que la etapa de más facilidad en el aprendizaje, es la evaluación, porque sólo hace referencia a que si se alcanzaron o no los objetivos trazados. A esto, podemos añadir lo dicho por los docentes:

“...la etapa más fácil del PAE, es la valoración, como tenemos ya la guía de recolección de datos, entonces nosotros les explicamos qué es lo que queremos que ellas investiguen, entonces se les da el formato, y ellas lo Ilenan..." (D8).

La mayoría (11/14) de las docentes entrevistadas afirman, que la valoración es la etapa del PAE más fácil de enseñar, esto debido a que las estudiantes cuentan con formatos de recolección de datos, técnicas como entrevista, examen físico, observación, que facilitan la obtención de datos; 1 / 14 afirma, que la etapa de más facilidad es la planificación, puesto que solo requiere de un manejo adecuado de la taxonomía NANDA, 
NIC, NOC; el mismo porcentaje (1/14) afirma que la etapa más fácil de enseñar es la evaluación, porque sólo consiste en saber si se ha logrado o no lo que se puso como objetivo; mientras que solo $1 / 14$ refiere que todas las etapas del PAE, son fáciles de enseñar y aprender.

Como podemos evidenciar el mayor porcentaje de docentes y la mitad de estudiantes entrevistados, coinciden en que la etapa del PAE, más fácil de enseñar y aprender es la valoración, misma que consiste en la obtención de datos significativos del estado de salud de una persona a partir de técnicas, como, la observación, la entrevista y, el examen físico céfalo-caudal 18 , siendo esta la primera fase y la más importante para aplicar un PAE efectivo, que satisfaga todas las necesidades afectadas de las persona cuidada.

\section{CATEGORÍA II: EXPERIENCIAS EN LA APLICACIÓN DEL PAE EN EL CONTEXTO HOSPITALARIO $Y$ COMUNITARIO}

Respecto a la aplicación del PAE en el contexto hospitalario, los estudiantes destacan lo siguiente:

“...en el campo hospitalario, la docente nos designaba un paciente a cada uno, evaluábamos con el examen físico, la observación y la entrevista, lo que nos permitía obtener todos los datos, sus antecedentes personales y familiares, para luego elaborar los diagnósticos y planes de cuidado, de acuerdo a las necesidades del paciente a cargo..." (E8).

Casi todos (15/16) los estudiantes refieren, que aplicaban el PAE en el ámbito hospitalario, el cual se iniciaba con la asignación de un paciente a cada estudiante, por parte de la docente tutora, luego se realizaba la recogida de datos a través de las diversas fuentes y técnicas de recolección, posteriormente, cada estudiante realizaba la validación de los datos significativos de su persona cuidado con las características definitorias de la NANDA, formulaban los diagnósticos enfermeros, si había disponibilidad de tiempo, la docente a cargo, realizaba una explicación acerca de la patología o necesidad alterada en el paciente, a modo de marco teórico, se establecían las intervenciones y actividades a realizar, luego de ejecutarlas, al final del turno, se realizaba la evaluación de las actividades ejecutadas y finalizaban con el registro de las notas de enfermería según el modelo SOAPIE (datos subjetivos, objetivos, análisis de datos, plan de atención, intervención, evaluación de resultados); y solo un mínimo porcentaje (1/16) refiere que en el ámbito hospitalario, simplemente se trata de modificar los diagnósticos médicos a diagnósticos enfermeros, a través de la NANDA. Al respecto las docentes manifestaron lo siguiente:

"...desde que se les asigna el paciente, las estudiantes empiezan a valorar, luego formulan sus diagnósticos, planifican, intervienen y evalúan, cuando ya están terminando hacen sus notas SOAPIE y con ello realizan la evaluación de los objetivos planteados y dejan evidencias del PAE aplicado..." (D7).

La mayoría de los discursos vertidos por las docentes, convergen con lo manifestado por los estudiantes, así pues $11 / 14$ docentes entrevistadas, refieren que en el ámbito hospitalario se da la aplicación del PAE, se inicia asignando a cada estudiante uno o más pacientes, ellos valoran y recogen los datos significativos, validan datos y formulan diagnósticos, luego establecen las intervenciones que van a realizar, las realizan y por último, evalúan su objetivo registrándolo en la nota de enfermería, y el resto $(3 / 14)$ de las docentes entrevistadas no enseñan ni aplican el PAE hospitalario, esto es debido a que dos de ellas trabajan en establecimientos de salud y sólo realizan la aplicación del PAE comunitario, y una docente no labora en ningún establecimiento de salud. 
Se trae a mención, entonces, a Pardo, quien, en su investigación, afirma que, la aplicación del proceso está limitada principalmente porque el personal de enfermería enmarca su trabajo en dar cumplimiento a las indicaciones médicas con pobre actuación independiente, y no ocupa en el equipo de salud el lugar que le corresponde, sino que la aplicación del PAE es parcial o casi nula por parte de las enfermeras 19.

Los estudiantes aplican el PAE en los contextos hospitalarios y comunitarios, adquieren experiencias con personas con problemas de salud, se relacionan, analizan, las cuidan; pero también tienen experiencias con personas sanas, $o$ en riesgo de padecer alguna enfermedad, en donde aplican el PAE involucrando a las personas para fomentar su propio cuidado:

“...en el ámbito comunitario, tenemos al paciente que llega a sus consultas en una posta, luego vamos haciendo el seguimiento más allá, vemos el lugar donde vive, los problemas y las necesidades que tenga, interactuamos más con la familia y con su comunidad, ya no vemos a la persona en sí, vemos su entorno..."

(E2).

Respecto al aprendizaje y aplicación del PAE en el ámbito comunitario, todos los estudiantes refieren que han aplicado por lo menos una vez el PAE en la comunidad, sea en un centro de salud o mediante las visitas domiciliarias, se les asignaba un paciente $o$ una familia, se establecían acuerdos para la realización de la visita domiciliaria, se recogían datos y se planificaba para realizar las intervenciones, las cuales estaban mayormente dirigidas a la promoción de la salud y prevención de la enfermedad.

Asimismo, algunas de las docentes refieren:

“...se le asigna un caso, de acuerdo a eso, se les va orientando a cómo van a presentarlo, qué es lo que van a incluir en su proceso, dentro de la planificación están las visitas domiciliarias, se trabaja con la familia, hacen su valoración de todo y luego hacen sus diagnósticos, hacen también sus sesiones educativas basados en la prevención..." (D2).

De las docentes entrevistadas, un 43\% (6), refieren que sí se realiza la enseñanza y aplicación del PAE en el ámbito comunitario, estas docentes laboran en establecimientos de salud o realizan las prácticas comunitarias, en donde inician con la asignación de familia o persona, realizan la visita domiciliaria y se ejecutan actividades en base a la promoción de la salud y prevención de enfermedades; y el otro $57 \%$ (8) de las docentes entrevistadas no enseñan ni aplican el PAE comunitario, esto es debido a que dichas docentes laboran sólo en instituciones hospitalarias, además de la universidad donde se realiza la investigación.

\section{CATEGORÍA III: EXPERIENCIAS EN LA EVALUACIÓN DE LA ENSEÑANZA DEL PAE}

Heather20, señala que es imprescindible establecer currículos comunes en las universidades con un enfoque enfermero $y$ desarrollar criterios adecuados para establecer el perfil de los tutores de prácticas con una evaluación continua de las competencias.

En la escuela de enfermería de la universidad donde se realizó la investigación, se aplican diversos formatos de evaluación de acuerdo a las asignaturas de especialidad, los formatos coinciden en su estructura al componerse de dos partes, la primera donde se evalúa la aplicación del PAE en un sujeto de cuidado a través de un seguimiento, denominado asesorías, y la segunda que evalúa el acto de sustentación del informe de la aplicación del PAE:

“...te evalúan mientras vas avanzando, a través de las asesorías, siempre te dan sugerencias, te van guiando por etapas y te van corrigiendo, incluso mientras acabaste, y cuando sustentas también te dicen, en qué estás fallando..." (E2). 
Los estudiantes refieren que la evaluación de la enseñanza del PAE es constante en la práctica clínica, y se da mediante las asesorías, cada docente de la primera rotación práctica, está a cargo de un grupo de estudiantes que pueden ser 5 o 6, dependiendo del número de estudiantes, la docente realiza la enseñanza y evaluación del PAE, que mayormente se da por etapas, los estudiantes realizan y presentan sus avances y las docentes realizan la revisión, que puede ser de manera personalizada o de manera virtual, como refieren algunas estudiantes.

Se añade a esto, lo referido por las docentes:

“...el PAE se evalúa con un instrumento que tiene dos partes, la primera parte es la evaluación que hace el docente asesor de acuerdo a los avances y asesorías que haya tenido el estudiante, donde se asigna una nota; la segunda parte contiene la nota de sustentación con criterios de evaluación sobre el contenido, la forma, el tono de voz, etc.; y con todo ello se obtiene la calificación final del PAE..." (D5)

Los discursos dados por las docentes convergen con los discursos dados por los estudiantes, todas las docentes refieren que la evaluación del PAE se realiza constantemente en la práctica clínica y mediante las asesorías. Asimismo, al realizar la presentación y sustentación del informe del PAE, las estudiantes refieren:

“...en un inicio tenía nervios, porque era la primera vez que iba a sustentar un PAE, ante el alumnado $y$ ante el jurado asignado, $y$ también tenía poca experiencia y había el miedo a ser descalificada, cada alumna realizaba su exposición sobre su caso clínico, las docentes jurado realizaban preguntas acerca del caso clínico, ellas se valían de una ficha de evaluación, que tenía un puntaje asignado, consistía en evaluar desde la presentación del alumno, y mayormente evaluaban los conocimientos que tenías del caso clínico..."
El $100 \%$ de los estudiantes entrevistadas refieren ser evaluadas mediante un formato de evaluación que incluye criterios de evaluación del seguimiento del PAE (asesorías), y criterios de evaluación de la sustentación del informe del PAE, asimismo, el 56\% (9) de los estudiantes refiere que durante la sustentación atraviesan por un periodo de miedo o estrés, debido a que les genera temor ser evaluados por más de una docente y, les preocupa qué interrogantes se les realizará; el $44 \%$ (7) de los estudiantes, sólo describieron el proceso de sustentación y de evaluación de la misma.

A ello, se añade uno de los discursos dados por las docentes:

“...en la sustentación, evaluamos con un formato en donde hay dos partes, la primera parte es en base a la evaluación del proceso en sí en forma física, y la segunda parte es ya la sustentación, que evalúa tono de voz, los conocimientos, si sustenta con seguridad, la posición que toma para la exposición, las diapositivas para la presentación, que no las lea, luego hacemos las preguntas al estudiante acerca del sujeto que estuvo a su cuidado..." (D8).

Los discursos de las docentes convergen con los discursos de los estudiantes. Todas las docentes afirman que durante la sustentación del informe del PAE, las docentes califican con un formato de evaluación que incluye criterios de evaluación de las asesorías del PAE y criterios de evaluación del acto de la sustentación, asimismo, algunas de las docentes, resaltaron la etapa de temor y estrés que atraviesan algunas estudiantes.

González y Chávez, en su investigación, mencionaron que una de las actividades que se realizan dentro de la práctica clínica es la elaboración y sustentación en grupo de un caso clínico real, seleccionado de los pacientes asignados en la rotación, donde el docente retroalimenta el proceso en cada uno de los pasos del PAE. La evaluación formativa la realiza 
el docente a diario, indicando al estudiante sus logros y dificultades tanto en lo personal como en lo profesional, a fin de buscar estrategias que le permitan superarse 5 .

\section{CATEGORÍA IV: ADQUISICIÓN DE HABILIDADES EN EL PROCESO DE ENSEÑANZA-APRENDIZAJE DEL PAE}

Para utilizar de manera efectiva el proceso de atención de enfermería, la enfermera debe poseer y ser capaz de aplicar algunas habilidades básicas. Se requiere de creatividad para la aplicación del conocimiento de enfermería, al igual que adaptabilidad para manejar los numerosos cambios inesperados que pueden presentarse. Además, son esenciales algunas habilidades interpersonales bien desarrolladas y técnicas competentes apropiadas 1 .

Las estudiantes a lo largo de su formación profesional y al aplicar el proceso de atención de enfermería, adquieren y desarrollan habilidades cognitivas, humanas y personales, que junto con el conocimiento y sustento científico, permiten que el cuidado a brindar sea óptimo, de calidad y competente:

"...he adquirido habilidad sobre todo en la búsqueda de la información, a la hora de fundamentar cada una de tus actividades, he aprendido a ver cuál es el fundamento de tu actividad, otra habilidad, es que cuando veo un signo y síntoma, o algo alterado, inmediatamente veo el diagnóstico de enfermería que le pertenece al paciente, rápidamente lo relacionas, más o menos qué diagnóstico puedes aplicar al paciente en tu proceso..." (E7).

El 56\% (9) de los estudiantes, refieren que durante la aplicación del PAE adquirieron habilidades cognitivas, aprendieron a realizar una valoración que incluya el uso de todas las técnicas, sobre todo desarrollaron la observación, asimismo, aprendieron a identificar de manera más rápida los diagnósticos de enfermería según NANDA a partir de los signos, síntomas, y dominios alterados en la persona, aprendieron también, a planificar y ejecutar intervenciones que ayuden de manera óptima a la persona cuidada, también refirieron adquirir mayor manejo de la taxonomía NANDA, NIC, NOC; y la más importante, que es la habilidad de adquirir más conocimiento al aplicar el PAE.

El otro porcentaje restante de estudiantes refieren:

“...he adquirido habilidades de cómo poder relacionarme más rápido con el paciente, porque para el PAE tienes que relacionarte bastante con el paciente, causar empatía, confianza, establecer una comunicación efectiva..." (E6).

El 44\% (7) de los estudiantes, refieren haber adquirido habilidades sociales, habilidades de interrelación, aprendieron a ser más comunicativas, a crear un ambiente de confianza, a entablar una conversación con el paciente, con sus familiares, a ser empáticas, a cuidar de las personas en todas sus dimensiones, incluyendo la emocional y psicosocial.

Esta información converge con los discursos referidos por las docentes respecto a la adquisición de habilidades durante el proceso de enseñanza-aprendizaje del PAE:

“..las estudiantes tienen habilidades para hacer una buena observación, para realizar un examen físico, para la fundamentación, eso significa, que llevan ciertos conocimientos, porque ya van a aplicar el fundamento científico para el cuidado..." (D3).

El $57 \%$ (8) de las docentes refieren que los estudiantes adquieren habilidades cognitivas en el proceso de aprendizaje del PAE, aprenden a valorar correctamente, a identificar necesidades alteradas y formular diagnósticos, a planificar intervenciones y fundamentar su acción, y a tener manejo de la taxonomía NANDA, NIC, NOC. 
Otro porcentaje de las docentes refieren:

“...las estudiantes tienen sensibilidad para empatizar con la persona cuidada, también para establecer relaciones positivas para poder aconsejar al familiar, otra habilidad, es la habilidad social, se relacionan fácilmente con el equipo de salud, también algunas son creativas para educar, realizan diferentes instrumentos, rotafolios, collage..." (D4).

El otro 43\% (6) de las docentes, refieren que los estudiantes adquieren habilidades sociales, entre ellas destacan, la empatía y confianza que entablan con el paciente, la creatividad que usan para elaborar el material con el que educarán al paciente y a sus familiares, la relación que establecen con el personal de salud, y también señalan el uso de la tecnología en la aplicación del PAE, al utilizar la taxonomía NANDA, NIC, NOC virtual durante las prácticas hospitalarias.

Según Pérez, el PAE exige del profesional: habilidades y destrezas efectivas, cognoscitivas $y$ motrices para observar, valorar, decidir, realizar, evaluar e interactuar con otros profesionales de la salud. De allí la importancia de que los futuros profesionales de enfermería, logren dichas competencias durante su formación profesional $^{21}$.

\section{CATEGORÍA V: NECESIDAD DE ESTANDARIZAR CRITERIOS EN LA ENSEÑANZA DEL PAE}

La enseñanza del PAE en la universidad donde se realizó la investigación necesita estandarizarse, se deben unificar criterios, de tal manera, que la enseñanza sea percibida por los estudiantes sin que se generen confusiones por la diversidad de metodologías empleadas.

Respecto a ello, se obtuvieron los siguientes discursos:

“...necesitamos que todas las docentes enseñen el PAE de la misma manera, a veces nos confundimos por la distinta metodología que usan al enseñarnos, y creemos que lo que estamos haciendo está mal; también, que los profesores se centren muy bien en las asesorías, porque hay algunos ciclos en los que tú sola haces el PAE, sin la ayuda de ninguna profesora..." (E2).

Dentro de las sugerencias aportadas por los sujetos de estudio: estudiantes, resalta que el $50 \%$ (8) de ellos, refieren que se necesita uniformizar criterios en la enseñanza del PAE, porque la enseñanza variada respecto a la taxonomía NANDA, NIC, NOC, genera confusión en el estudiante; un 19\% (3) de los estudiantes refieren que también se necesita asesoría personalizada, más que virtual, esto ayuda a resolver sus dudas y genera mejor comprensión de las correcciones dadas; un 25\% (4) de los estudiantes refieren que la enseñanza debe ser más didáctica, el docente debe ser más empático y mostrar seguridad para saber llegar al estudiante; y un 6\% (1) refiere que se debe dar mayor tiempo para realizar la valoración de la persona cuidada.

Se añade a esto, lo referido por los docentes:

"...la primera sugerencia es que los profesores tomemos la decisión de aplicar el $P A E$ en forma uniforme, si lo vamos a hacer por la valoración por dominios, utilizando la taxonomía NANDA-NIC-NOC, entonces que sea uniforme la aplicación, porque los estudiantes son los que van a estar en espera de eso, si eso no sucede se genera confusión en el estudiante, no sabe si aplicar NANDA o crear él sus diagnósticos..." (D10).

Dentro de las sugerencias aportadas por las docentes, resalta que el 65\% (9) de las docentes, refieren necesidad de la uniformidad en la enseñanza del PAE, sobre todo al utilizar la taxonomía NANDA, NIC, NOC, para evitar que se generen dudas en los estudiantes, y lograr dominio en el conocimiento.

Otras docentes refieren: 
“...una sugerencia a las docentes, sería que procuremos exigir a las estudiantes un poquito más, porque a veces si no les exigimos no avanzan, y a las estudiantes sería que investiguen más, tengan más literatura para fundamentar bien sus cuidados..." (D8).

Un 28\% (4) sugieren mayor exigencia a las demás docentes en la asesoría y evaluación del PAE, asimismo sugieren que el estudiante tenga mayor responsabilidad en el cumplimiento de sus avances; y un 7\% (1) de las docentes, sugiere que las docentes deben tener mayor empatía con el estudiante e inspirarle confianza.

\section{Conclusiones}

Los estudiantes de enfermería, al iniciar el aprendizaje del Proceso de Atención de Enfermería (PAE), presentan dificultad al relacionarse con la taxonomía NANDA, NIC, NOC; debido a que no existe una uniformidad en la enseñanza del mismo, se puede rescatar que la enseñanza del PAE se da por medio de la estrategia de casos clínicos y de manera personalizada. EI PAE, es aplicado en contextos hospitalarios y comunitarios. Respecto al primero, las estudiantes están a cargo de al menos un paciente, a quien deben aplicar el PAE con supervisión de la docente; en este ámbito, se presenta como principal limitación, el poco conocimiento y análisis crítico que muestran los estudiantes al realizar la etapa de diagnóstico. Respecto al segundo contexto, el comunitario, los estudiantes refieren haber tenido poca experiencia al aplicar el PAE en este ámbito.

La evaluación de la enseñanza del PAE se realiza principalmente con un formato de evaluación, que contiene dos partes; la primera parte, evalúa el seguimiento y las asesorías que el docente brinda de acuerdo a los avances del informe del PAE que presenta el estudiante, se refiere que, en algunas oportunidades, estas asesorías se brindan de manera virtual, siendo un poco difícil su comprensión, y que es mejor recibir y brindar una asesoría personalizada. La segunda parte, evalúa el acto de sustentación del informe del PAE, ante el cual los estudiantes atraviesan una etapa de estrés y temor, siendo muy valioso la evaluación asertiva de los docentes que forman parte del jurado evaluador.

Por otro lado, el aprendizaje y aplicación del PAE, genera en el estudiante el descubrimiento $y$ desarrollo de habilidades, que pueden ser cognitivas o también habilidades sociales; se rescata también que existe una necesidad urgente de uniformizar los criterios en la enseñanza del PAE, para evitar confusiones y dudas en los estudiantes, debido a la variación en la metodología y criterios de enseñanza; a la ausencia de asesorías personalizadas en algunos casos, ya que estas se realizan de manera virtual, lo cual genera insatisfacción en los estudiantes y por tanto un aprendizaje del PAE, deficiente.

\section{Limitaciones del estudio:}

Las investigadoras declaran haber tenido ciertas dificultades en la recolección de datos, tales como: limitación para acceder a las docentes de prácticas, puesto que no asisten a la universidad y solo se les podía ubicar en su centro asistencial, lo que requirió de mayor tiempo para la recogida de datos, lo que inicialmente estaba propuesto en un mes, se hizo en dos. Igualmente, para realizar el chequeo de participantes que estaba contemplado realizarse de manera personal, algunas se tuvieron que hacer de manera virtual, vía email.

\section{Responsabilidades éticas}

Protección de personas y animales. Los autores declaran que para esta investigación no se han realizado experimentos en seres humanos ni en animales.

Confidencialidad. Los autores declaran que han seguido los protocolos de su centro de estudios sobre la publicación de datos de los participantes del estudio. 
Se obtuvo el respectivo consentimiento informado y en el presente artículo no aparecen datos de los participantes previo consentimiento informado; el tamaño de la muestra se determinó por la técnica de saturación y redundancia. Para asegurar la confidencialidad, de los enfermeros y madre de familia se usó códigos de identificación, para las madres: MADR1, MADR2...MADR15, y a las enfermeras ENF1, ENF2 ......ENF1 0.

Conflicto de intereses: Los autores declaran no tener conflictos de intereses.

Financiamiento: Ninguno.

\section{Bibliografía}

1. Doenges $M$, Frances $M$. Proceso $y$ Diagnósticos de Enfermería. Aplicaciones. Bogotá: El Manual Moderno; 2014. 319 p.

2. Díaz $\mathbf{W}$, Nieto $C$. Utilización del proceso de enfermería en el ejercicio clínico profesional en seis instituciones prestadoras de servicios de salud en Santafé de Bogotá. Rev Actualiz Enf [Internet]. Jun 2000. [Citado el 12 de setiembre de 2018]; 3 (2): 10-11. Disponible en:

https://encolombia.com/medicina/revistasmedicas/enfermeria/ve-

32 /enfermeria3200-utilizacion/

3. Alfaro R. Aplicación del proceso enfermero. Fundamento del razonamiento crítico. $8^{\mathrm{a}}$ ed. Barcelona: Wolters Kluwer; 2014. 36 p.

4. Becerra A, La Serna K. Diseño curricular por competencias: Un enfoque para carreras del campo económico-empresarial. Lima: Universidad del Pacífico; 2016. 137 p.

5. Gonzáles P, Chávez A. Proceso de atención de enfermería desde la perspectiva docente. Inv en Enf: Imagen y Desarrollo. Colombia [Internet]. Dic 2009 [Citado el 21 de setiembre de 2016]; 11 (2): 47-50. Disponible en: https://revistas.javeriana.edu.co/index.php/ imagenydesarrollo/article/view/1617
6. Dolores C. Nivel de aplicación del proceso de atención de enfermería por los internos de enfermería de la UNMSM. Lima; 2005. 57 p.

7. Hernández R, Fernández $C$, Baptista $P$. Metodología de la investigación. Nuevo York: McGraw-Hill Education; 2018. 714 p.

8. Burns N, Grove S. Investigación en Enfermería. 6ta ed. España: Elsevier; 2016. $554 p$.

9. Palomino J, Peña J, Zevallos G, Orizano L. Metodología de la Investigación: Guía para elaborar un proyecto en Salud y Educación. Lima: San Marcos; 2017. 223p.

10. Bernal C. Metodología de la investigación. 4ta ed. Colombia: Pearson; 2016. 286p.

11. Ñaupas $H$, Valdivia $M$, Palacios J, Romero $H$. Metodología de la Investigación CuantitaivaCualitativa y Redacción de la Tesis. $5^{a}$ ed. Bogotá: Ediciones de la U; 2018. 559p.

12. Sgreccia E. Manual de bioética. La bioética y sus principios. 4th ed. Madrid: Series BAC Maior; 2014. 877p.

13. Noreña A, Alcaraz N, Rojas J, Rebolledo D. Aplicabilidad de los criterios de rigor y éticos en la investigación cualitativa. Rev Actualiz Enf [Internet]. May 2012 [Citado el 15 de octubre. de 2018]; 12 (3): 263-74. Disponible en:

http://www.scielo.org.co/pdf/aqui/v12n3/v 12n3a06

14. Iyer P, Taptich B, y Bernocchi D. Proceso de enfermería y diagnósticos de enfermería. 3ra ed. Madrid: McGraw-Hill Interamericana Editores; $1997.111 \mathrm{p}$.

15. Cachón J, Álvarez C, Palacios D. El significado del lenguaje estandarizado NANDA-NIC-NOC en las enfermeras de cuidados intensivos madrileñas: abordaje fenomenológico. Enferm Intensivista [Internet]. Abr 2016 [Citado 20 oct 2018]; 23(2): 68-76. Disponible en:

https://www.picuida.es/el-significado-dellenguaje-estandarizado-nanda-nic-noc-enlas-enfermeras-de-cuidados-intensivosmadrilenas-abordaje-fenomenologico/ 
16. Dirección de Investigación y Desarrollo Educativo de Monterrey. El estudio de casos como técnica didáctica. Las estrategias y técnicas didácticas en el rediseño [Internet]. México: Dirección de Investigación y Desarrollo Educativo de Monterrey [Citado 20 oct 2018]. Disponible en: http:// sitios.itesm.mx/va/dide2/tecnicas_di dacticas/casos/casos.pdf

17. Accornero P, Santucho E. Proyecto de investigación: Dificultades en el aprendizaje del Proceso de atención de enfermería en los estudiantes del primer ciclo de la carrera Licenciatura en Enfermería. [Tesis de Licenciatura en internet]. Córdova: Universidad Nacional de Córdova; 2011 [Citado 18 jun 2020]. 85 p. Disponible en: https://es.scribd.com/document/32048466 7/accornero-pablo-pdf

18. Reina N. El Proceso de Enfermería: Instrumento para el cuidado. Umbral Científico Redalyc. [Internet]. Colombia. 2010 [Citado el 09 de junio. de 2018]; 17: 18-23. Disponible en: http: / / www.redalyc.org/articulo.oa?id = 3042 1294003

19. Pardo M. Factores que limitan a las enfermeras la aplicación del proceso de atención de enfermería según percepción de los internos de la escuela académico profesional de enfermería de la UNMSM. Lima; $2013.79 \mathrm{p}$.

20. Heather T, Weir D, Monteiro D, Moorhead S, Butcher H, Martín S. II Congreso Latinoamericano NANDA Internacional 2015. Evolución del Cuidado de Enfermería desde el Lenguaje Estandarizado NANDA NIC NOC. Colombia. 2015

21. Pérez M. Operacionalización del proceso de atención de enfermería. Rev Mex Enf Card. [Internet]. Agos 2002. [Citado el 12 Set 2018]; 10(2): 62-6. Disponible en: http://www.medigraphic.com/pdfs/enfe/en -2002 /en022d.pdf 